\title{
Surgery for Bismuth-Corlette Type 4 Perihilar Cholangiocarcinoma: Results from a Western Multicenter Collaborative Group
}

\begin{abstract}
Andrea Ruzzenente, MD, PhD ${ }^{1}$, Fabio Bagante, $\mathrm{MD}, \mathrm{PhD}^{1}{ }^{1}$, Pim B. Olthof, $\mathrm{MD}, \mathbf{P h D}^{2,3}$, Luca Aldrighetti, $\mathrm{MD}, \mathrm{PhD}^{4}$, Ruslan Alikhanov, $\mathrm{MD}, \mathrm{PhD}^{5}$, Matteo Cescon, $\mathrm{MD}$, $\mathrm{PhD}^{6}$, Bas Groot Koerkamp, $\mathrm{MD}$, $\mathrm{PhD}^{2}$, William R. Jarnagin, $\mathrm{MD}^{7}$, Silvio Nadalin, $\mathrm{MD}^{8}$, Johann Pratschke, MD, PhD ${ }^{9}$, Moritz Schmelzle, MD ${ }^{9}$, Ernesto Sparrelid, MD, PhD ${ }^{10}$, Hauke Lang, $\mathrm{MD}^{11}$, Calogero Iacono, $\mathrm{MD}^{1}$, Thomas M. van Gulik, MD, $\mathrm{PhD}^{3}$, Alfredo Guglielmi, MD ${ }^{1}$, and Perihilar Cholangiocarcinoma Collaboration Group

${ }^{1}$ Department of Surgery, Unit of Hepato-Pancreato-Biliary Surgery, University of Verona Medical School, Verona, Italy; ${ }^{2}$ Department of Surgery, Erasmus Medical Center, Rotterdam, The Netherlands; ${ }^{3}$ Department of Surgery, Amsterdam University Medical Center, University of Amsterdam, Amsterdam, The Netherlands; ${ }^{4}$ Hepato-biliary Surgery Division, Ospedale San Raffaele-IRCCS, Milan, Italy; ${ }^{5}$ Department of Hepato-Pancreato-Biliary Surgery, Moscow Clinical Scientific Center, Moscow, Russia; ${ }^{6}$ Department of Medical and Surgical Sciences, S. Orsola-Malpighi Hospital, Alma Mater Studiorum - University of Bologna, Bologna, Italy; ${ }^{7}$ Division of Hepatopancreatobiliary Surgery, Memorial SloanKettering Cancer Center, New York, NY; ${ }^{8}$ Department of General and Transplant Surgery, University Hospital Tübingen, Tübingen, Germany; ${ }^{9}$ Department of Surgery, Charité - Universitätsmedizin Berlin, Berlin, Germany; ${ }^{10}$ Department of Surgery, Centre for Digestive Diseases, Karolinska University Hospital, Stockholm, Sweden; ${ }^{11}$ Department of General, Visceral and Transplantation Surgery, University Hospital of Mainz, Mainz, Germany
\end{abstract}

\begin{abstract}
Background. Although Bismuth-Corlette (BC) type 4 perihilar cholangiocarcinoma (pCCA) is no longer considered a contraindication for curative surgery, few data are available from Western series to indicate the outcomes for these patients. This study aimed to compare the short- and long-term outcomes for patients with BC type 4 versus $\mathrm{BC}$ types 2 and 3 pCCA undergoing surgical resection using a multi-institutional international database.
\end{abstract}

The members of the Perihilar Cholangiocarcinoma Collaboration Group have been listed in acknowledgements.

Supplementary Information The online version contains supplementary material available at https://doi.org/10.1245/s10434021-09905-z.

(C) The Author(s) 2021

First Received: 26 October 2020

Accepted: 11 March 2021;

Published Online: 6 May 2021

C. Iacono, MD

e-mail: Calogero.Iacono@univr.it
Methods. Uni- and multivariable analyses of patients undergoing surgery at 20 Western centers for BC types 2 and 3 pCCA and BC type 4 pCCA.

Results. Among 1138 pCCA patients included in the study, $826(73 \%)$ had BC type 2 or 3 disease and 312 (27\%) had type 4 disease. The two groups demonstrated significant differences in terms of clinicopathologic characteristics (i.e., portal vein embolization, extended hepatectomy, and positive margin). The incidence of severe complications was $46 \%$ for the BC types 2 and 3 patients and $51 \%$ for the BC type 4 patients $(p=0.1)$. Moreover, the 90 -day mortality was $13 \%$ for the $\mathrm{BC}$ types 2 and 3 patients and $12 \%$ for the BC type 4 patients $(p=$ 0.57). Lymph-node metastasis ( $\mathrm{N} 1$; hazard-ratio [HR], 1.62), positive margins (R1; HR, 1.36), perineural invasion (HR, 1.53), and poor grade of differentiation (HR, 1.25) were predictors of survival (all $p \leq 0.004$ ), but BC type was not associated with prognosis. Among the N0 and R0 patients, the 5-year overall survival was $43 \%$ for the patients with BC types 2 and 3 pCCA and $41 \%$ for those with $\mathrm{BC}$ type 4 pCCA $(p=0.60)$.

Conclusions. In this analysis of a large Western multiinstitutional cohort, resection was shown to be an acceptable curative treatment option for selected patients with BC 
type 4 pCCA although a more technically challenging surgical approach was required.

Perihilar cholangiocarcinoma (pCCA) is a rare tumor originating from the epithelium of the hepatic duct confluence and commonly classified according to the BismuthCorlette classification (BC) based on the extent of proximal biliary infiltration. ${ }^{1}, 2$ In particular, pCCA bilaterally involving the second-order biliary ducts has been classified as $\mathrm{BC}$ type 4. According to several prognostic staging systems (e.g., the American Joint Committee on Cancer [AJCC]-TNM 7th edition) as well as the American Hepato-Pancreato-Biliary Association (AHPBA) guidelines, BC type 4 has pCCA been considered a locally advanced tumor amenable to surgical treatments only for highly selected patients. ${ }^{3}, 4$

Moreover, surgical resection of BC type 4 pCCA often requires extended hepatectomies (i.e., trisectionectomy), intrahepatic bile duct reconstruction, lymphadenectomy, and vascular resections that in addition to the innate technical challenges have been associated with a high risk of postoperative complications and mortality. ${ }^{5} 6$ Although BC type 4 therefore has represented a criterion for unresectability, recent experiences in Eastern and Western series have suggested that surgical resection with curative intent (i.e., negative margin [R0]) might offer a valid treatment option for this disease stage. ${ }^{7}$

Because recent improvements in surgical techniques and perioperative management of patients undergoing major liver surgery (i.e., extended hepatectomies with $\geq 5$ liver segments) have reduced the rates of postoperative morbidity and mortality, several authors have currently supported resection with curative intent for patients with BC type 4 pCCA. In addition, instead of BC types, several variables, including surgical margin and lymph node status, have been associated with oncologic outcomes for pCCA patients undergoing surgery. ${ }^{12}, 13$ For these reasons, in the new eighth edition of the AJCC TNM staging system, BC type 4 was removed as a criterion from the definition of stage T4 disease. ${ }^{4}$

To date, only few data have compared the short-term (i.e., morbidity and mortality) and long-term (i.e., overall and disease-free survival) outcomes of liver surgery with radical intent for BC type 4 tumors in the Western series. For these reasons, the current study, using a multi-institutional international database, aimed to assess the overall complications and mortality of $\mathrm{BC}$ type 4 pCCA treated with curative-intent surgical resection compared with those of BC types 2 and 3 pCCA. In addition, oncologic outcomes, including overall and disease-free survival, were compared.

\section{PATIENTS AND METHODS}

Patients who underwent surgical resection at one of the 20 tertiary referral hepato-biliary Western centers were included in the study (Supplementary Material). Participating centers had a median of 80 (range 25-115) consecutive resections for pCCA between January 2000 to December 2017. The study defined pCCA as a biliary tumor involving the hepatic duct confluence according to the definition of the Japanese Society of Biliary Surgery (JSBS). ${ }^{14}$ The study excluded all patients who underwent explorative surgery only, resection with macroscopic residual tumor ( $\mathrm{R} 2$ ), or excision of only the extra-hepatic bile ducts or transplantation, as well as patients with metastases or distant lymph-node metastases (N2). Furthermore, patients with BC type 1 pCCA were excluded from the study given the relative heterogeneity in the surgical approach for this type of pCCA (i.e., bile duct resection only). The institutional review board of the participating institutions approved the study.

The multicenter design of the current study clearly implied differences in the preoperative management of the included patients. The type of preoperative evaluation of liver function varied among the different centers according to surgeon and center preferences, whereas future remnant liver volume (FRLV) was evaluated with computed tomography (CT) volumetry. Therefore, the selection of patients for preoperative portal vein embolization (PVE) and biliary drainage differed between centers according to center and surgeon preferences. In general, most patients for whom major liver resections were planned underwent preoperative, endoscopic, or transhepatic biliary drainage of at least the future remnant liver.

Preoperative cholangitis was defined as fever and leukocytosis requiring (additional) biliary drainage in accordance with the definitions applied in the DROP and DRAINAGE trials dealing with preoperative biliary drainage. $^{15}, 16$ Bismuth-Corlette (BC) classification was determined using preoperative imaging studies including contrast-enhanced CT, magnetic resonance imaging (MRI), MR cholangiography, and when available, endoscopic retrograde cholangiopancreatography (ERCP). BismuthCorlette type 4 pCCA was defined as tumor infiltration extending proximally into the segmental biliary ducts on both sides of the liver, usually including segment 1 . The BC classification was locally assessed and then surgically confirmed after the surgical operation. Type of surgery, histologic data, and postoperative outcomes were recorded and analyzed in the collective database. Major and extended liver resections were defined as resections of at least three and five Couinaud liver segments, respectively. In the 
respective pathology reports, R0 resection was defined as tumor-free margins in all the reported margins (biliary and circumferential margins).

All complications after surgery were collected and classified according to the Clavien-Dindo classification system, with grade 3 or higher considered as major morbidity. ${ }^{17}$ Liver failure, biliary leakage, and hemorrhage were scored and classified according to the respective International Study Group of Liver Surgery (ISGLS) criteria, and only grades B and C were considered clinically relevant. ${ }^{18}-20$ Perioperative mortality was defined as death within 90 days after surgery.

\section{Statistical Analyses}

Continuous variables were reported as medians with interquartile ranges (IQRs), whereas categorical variables were reported as totals and frequencies. Comparisons between categorical variables were assessed using the chisquare test or Fisher's exact test, as appropriate. The outcomes for the survival analysis were overall survival (OS) and disease-free survival (DFS). The study defined OS as the interval between the date of surgery and the date of the patient's death and recurrence as the interval between the date of surgery and the date or recurrence.. At the date of the last follow-up visit, OS was censored for the patients who were alive, and DFS was censored for those who remained disease-free. Both OS and DFS were estimated by Kaplan-Meier methodology, and survival curves were compared using log-rank analysis.

Cox proportional hazards regression analysis was used to evaluate any association among variables and survival outcomes, with coefficients reported as hazard ratios (HRs) and corresponding 95\% confidence intervals (CIs). Variables with a $p$ value lower than 0.1 in univariable analysis were included in the final multivariable models. Akaike information criterion (AIC) model selection was used to distinguish among a set of possible models describing the relationship between OS (and DFS) and the variables with $p$ value lower than 0.1 in the univariable analysis. The bestfit model carrying the lowest AIC score was selected as the final model.

To account for potential residual confounders regarding the effect of surgical approach on outcomes, propensity scores were estimated using a logistic regression model with BC type as a dependent variable specified as type 4 versus types 2 and 3 pCCA. Gender, age, American Society of Anesthesiology (ASA) score, Ca 19-9 serum level, drainage, preoperative cholangitis, and PVE were independent variables in the logistic regression model. An exact propensity score value was used for matching. The degrees of covariate imbalance were measured using the standardized (mean and proportion) differences, as proposed by
Austin $^{21}$ A $p$ value lower than 0.05 (two-tailed) was considered statistically significant. All analyses were performed using STATA version 12.0 (StataCorp LP, College Station, TX, USA) and R version 3.6.1 (2019-0705) "Action of the Toes," with the additional packages (i.e., survival and matching packages). ${ }^{22}$

\section{RESULTS}

\section{Patients' Baseline Characteristics of BC Type 4 pCCA}

Among the 1138 patients who underwent surgery for pCCA, 312 (27.4\%) had BC type 4 tumors, whereas 826 $(72.6 \%)$ had BC type 2 or 3 tumors (Table 1$)$. The majority of the BC type 4 pCCA patients were male $(n=179$, $57.4 \%$ ), and about half of the patients were older than 65 years $(n=155,49.7 \%)$. Of the 284 BC type 4 patients (91\%) who required preoperative biliary drainage, $20.8 \%$ $(n=65)$ were treated with percutaneous transhepatic biliary drainage (PTBD) and 48.7\% ( $n=152)$ were treated with endoscopic biliary drainage. The vast majority of the patients $(97.1 \% ; n=303)$ underwent major hepatectomy ( $\geq 3$ liver segments), whereas 190 of the patients $(60.9 \%)$ underwent an extended liver resection ( $\geq 5$ liver segments).

The analysis included 9 rare cases of patients $(2.9 \%)$ who underwent central hepatectomy $(<3$ segments) and 113 cases of patients $(36.2 \%)$ who underwent left and right hemi-hepatectomy ( $<5$ segments). Interestingly, the incidence of positive margin (R1) did not differ between the two groups (R1: 2 [22.2\%] without major hepatectomy vs 120 [39.6\%] with major hepatectomy; $p=0.29$; R1: 54 [44.3\%] without extended hepatectomy vs 68 [35.8\%] with extended hepatectomy; $p=0.13$ )./I/I

Vascular resection/reconstruction was required for 163 patients $(52.2 \%)$ including 150 cases of portal vein resection/reconstruction only (48.1\%), 8 cases of hepatic artery resection/reconstruction only ( $2.5 \%)$, and 5 cases of combined portal vein and hepatic artery resection/ reconstruction $(1.6 \%)$. The overall incidence of severe complications (Clavien-Dindo $\geq 3$ ) was $51.3 \%(n=160)$, and the overall incidence of 90-day mortality was $12.2 \%$ $(n=38)$.

Among $61(19.6 \%)$ patients who had a PHLF, 11 $(18.0 \%, 11 / 61)$ had an associated intra-abdominal abscess. Moreover, 198 patients (17.4\%) had a median hospital stay (LoS) of 14.5 days (IQR, 10-24 days), with $71.4 \%$ ( $n=$ 223) having an LoS longer than 10 days. During a median follow-up period of 21 months, the median overall survival (OS) was 28.4 months (95\% CI, 23.7-33.0 months), and the median disease-free survival (DFS) was 26.3 months (95\% CI, 19.0-33.7 months). 
TABLE 1 Baseline characteristics of perihilar cholangiocarcinoma patients with Bismuth type $4(n=312)$ versus Bismuth type 2 or $3(n=826)$ disease

\begin{tabular}{|c|c|c|c|}
\hline Variables & $\begin{array}{l}\text { Bismuth type } 4 \\
n(\%)\end{array}$ & $\begin{array}{l}\text { Bismuth type } 2 \text { or } 3 \\
n(\%)\end{array}$ & $p$ value \\
\hline No. of patients & 312 & 826 & - \\
\hline Age (years) & & & 0.43 \\
\hline$\leq 65$ & $157(50.3)$ & $394(47.7)$ & \\
\hline$>65$ & $155(49.7)$ & $432(52.3)$ & \\
\hline Gender & & & 0.94 \\
\hline Male & $179(57.4)$ & $472(57.1)$ & \\
\hline Female & $133(42.6)$ & $354(42.9)$ & \\
\hline ASA physical status classification & & & 0.99 \\
\hline $1-2$ & $189(62.4)$ & $475(62.3)$ & \\
\hline $3-4$ & $114(37.6)$ & $287(37.7)$ & \\
\hline Preoperative biliary drainage & & & 0.001 \\
\hline No & $28(9.0)$ & $127(15.4)$ & \\
\hline Yes & $284(91.0)$ & $699(84.6)$ & \\
\hline Type of biliary drainage & & & $<0.001$ \\
\hline No drainage & $28(9.0)$ & $127(15.40)$ & \\
\hline PTBD & $65(20.8)$ & $238(28.9)$ & \\
\hline Endoscopic biliary drainage & $152(48.7)$ & $339(41.2)$ & \\
\hline Both & $67(21.5)$ & $119(14.5)$ & \\
\hline Preoperative cholangitis & & & 0.95 \\
\hline No & $237(77.5)$ & $600(77.8)$ & \\
\hline Yes & $69(22.5)$ & $171(22.2)$ & \\
\hline Portal vein embolization & & & $<0.001$ \\
\hline No & $230(73.7)$ & $681(82.4)$ & \\
\hline Yes & $82(26.3)$ & $145(17.6)$ & \\
\hline CA 19-9 ( U/mL) & & & 0.17 \\
\hline$\leq 100$ & $216(69.2)$ & $536(64.9)$ & \\
\hline$>100$ & $96(30.8)$ & $290(35.1)$ & \\
\hline Major liver resection ( $\geq 3$ segments) & & & 0.2 \\
\hline Yes & $303(97.1)$ & $812(98.3)$ & \\
\hline No & $9(2.9)$ & $14(1.7)$ & \\
\hline Extended liver resection ( $\geq 5$ segments) & & & $<0.001$ \\
\hline Yes & $190(60.9)$ & $383(46.4)$ & \\
\hline No & $122(39.1)$ & $443(53.6)$ & \\
\hline Type of liver resection & & & $<0.001$ \\
\hline Left hepatectomy & $66(21.1)$ & $286(34.6)$ & \\
\hline Extended left hepatectomy & $81(26.0)$ & $121(14.6)$ & \\
\hline Right hepatectomy & $47(15.1)$ & $143(17.3)$ & \\
\hline Extended right hepatectomy & 109 (34.9) & $262(31.8)$ & \\
\hline Segments 4 and 5 & $3(1.0)$ & $9(1.1)$ & \\
\hline Central hepatectomy & $6(1.9)$ & $5(0.6)$ & \\
\hline Caudate lobe resection & & & 0.25 \\
\hline Yes & $141(45.2)$ & $491(59.4)$ & \\
\hline No & $52(16.7)$ & $146(17.7)$ & \\
\hline NA & $119(38.1)$ & 189 (22.9) & \\
\hline Portal vein resection & & & $<0.001$ \\
\hline Yes & $155(49.7)$ & $244(29.5)$ & \\
\hline No & $157(50.3)$ & $582(70.5)$ & \\
\hline Hepatic artery resection & & & 0.16 \\
\hline Yes & $13(4.2)$ & $21(2.5)$ & \\
\hline No & $299(95.8)$ & 805 (97.5) & \\
\hline
\end{tabular}


TABLE 1 continued

\begin{tabular}{|c|c|c|c|}
\hline Variables & $\begin{array}{l}\text { Bismuth type } 4 \\
n(\%)\end{array}$ & $\begin{array}{l}\text { Bismuth type } 2 \text { or } 3 \\
n(\%)\end{array}$ & $p$ value \\
\hline Perineural invasion & & & 0.43 \\
\hline Yes & $204(65.4)$ & $532(64.4)$ & \\
\hline No & $57(18.3)$ & $176(21.3)$ & \\
\hline NA & $51(16.3)$ & $118(14.3)$ & \\
\hline Tumor grade & & & 0.61 \\
\hline Well/moderately & $222(71.2)$ & $610(73.8)$ & \\
\hline Poor/undifferentiated & $70(22.4)$ & $172(20.8)$ & \\
\hline NA & $20(6.4)$ & $44(5.4)$ & \\
\hline Surgical margins & & & 0.014 \\
\hline R0 & $190(60.9)$ & $567(68.6)$ & \\
\hline $\mathrm{R} 1$ & $122(39.1)$ & $259(31.4)$ & \\
\hline Lymphnodes status & & & 0.23 \\
\hline No & $170(54.5)$ & $483(58.5)$ & \\
\hline N1 & $142(45.4)$ & $343(41.5)$ & \\
\hline Median hospital stay: days (IQR) & $14.5(10.0-24.0)$ & $12.0(11.0-15.0)$ & $<0.001$ \\
\hline Post-hepatectomy liver failure & & & 0.25 \\
\hline No failure/PHLF A & $251(80.4)$ & $687(83.4)$ & \\
\hline PHLF B/C & $61(19.6)$ & $137(16.6)$ & \\
\hline Biliary leak & & & 0.1 \\
\hline No leak/grade A & $240(76.9)$ & $669(81.3)$ & \\
\hline Grade $\mathrm{B} / \mathrm{C}$ & $72(23.1)$ & $154(18.7)$ & \\
\hline Intraabdominal abscess $^{\mathrm{a}}$ & & & 0.16 \\
\hline No & $291(93.3)$ & 749 (90.7) & \\
\hline Yes & $21(6.7)$ & $77(9.3)$ & \\
\hline Severe bleeding complications ${ }^{\mathrm{a}}$ & & & 0.3 \\
\hline No & $301(96.5)$ & $785(95.0)$ & \\
\hline Yes & $11(3.5)$ & $41(5.0)$ & \\
\hline Other severe complications ${ }^{\mathrm{a}}$ & & & 0.3 \\
\hline No & $264(84.6)$ & $713(86.3)$ & \\
\hline Yes & $48(15.4)$ & $113(13.7)$ & \\
\hline Clavien-Dindo $\geq 3$ complications & & & 0.099 \\
\hline No & $152(48.7)$ & $446(54.2)$ & \\
\hline Yes & $160(51.3)$ & $377(45.8)$ & \\
\hline No. of severe complications ${ }^{\mathrm{a}}$ & & & 0.3 \\
\hline 1 & $131(81.9)$ & $283(75.5)$ & \\
\hline 2 & $24(15.0)$ & $76(50.5)$ & \\
\hline 3 & $5(3.1)$ & $12(3.2)$ & \\
\hline 4 & - & $3(0.8)$ & \\
\hline 30-Day mortality & & & 0.36 \\
\hline No & $284(91.0)$ & 767 (92.9) & \\
\hline Yes & $28(9.0)$ & $59(7.1)$ & \\
\hline 90-Day mortality & & & 0.57 \\
\hline No & $274(87.8)$ & $715(86.6)$ & \\
\hline Yes & $38(12.2)$ & $111(13.4)$ & \\
\hline
\end{tabular}

ASA, American Society of Anesthesiologists; PTBD, percutaneous transhepatic biliary drainage; NA, not available; IQR, interquartile range; PHLF, posthepatectomy liver failure

${ }^{\mathrm{a}}$ Clavien-Dindo $\geq 3$ complications 
Comparison of BC Type 4 With BC Types 2 and 3 $p C C A$

The patients with $\mathrm{BC}$ type 4 and those with $\mathrm{BC}$ type 2 or 3 pCCA demonstrated differences in terms of baseline clinical and pathologic characteristics (Table 1). In particular, the two groups, differed significantly in the use of PVE (BC type 4: $n=82$ [26.3\%]; BC type 2 or $3: n=145$ [17.6\%]; $p<0.001$ ), extended liver resection (BC type 4: $n=190[60.9 \%$ ]; BC type 2 or $3, n=383$ [46.4\%]; $p<$ 0.001 ), and positive margin status (R1; BC type $4, n=122$ [39.1\%]; BC type 2 or 3, $n=259$ [31.4\%]; $p=0.014)$. Conversely, the BC type 4 patients and the BC types 2 and 3 patients had comparable incidences of severe complications (Clavien-Dindo $\geq 3$ ) (BC type 4, $n=160$ [51.3\%]; BC types 2 and 3, $n=377$ [45.8\%]; $p=0.099$ ) and 90-day mortality (BC type 4, $n=38$ [12.2\%]; BC types 2 and 3, $n=111$ [13.4\%]; $p=0.574$ ) (Table 1). In particular, the BC type 4 patients and the BC types 2 and 3 patients had similar incidences of PHLF, biliary leak, intra-abdominal abscess, severe bleeding, other severe complications, and number of severe complications (all $p>0.1$; Table 1).

\section{Overall Survival Analysis of the pCCA Patients}

To investigate the oncologic outcomes for the BC type 4 patients and the BC types 2 and 3 patients, uni- and multivariable survival analyses were performed. The two groups had comparable OS $(p=0.063)$ and DFS $(p=0.94)$, with a median OS of 28.4 months (95\% CI, 23.7-33.0 months) for the BC type 4 patients and 33.8 months $(95 \%$ CI, 30.0-37.7 months) for the BC types 2 and 3 patients (Fig. 1). Similarly, the median DFS was 26.3 months $(95 \%$ CI, 19.0-33.7 months) for the BC type 4 patients and 29 months (95\% CI, 24.2-31.0 months) for the BC types 2 and 3 patients.

In the univariable survival analysis, several factors were associated with OS including PVE (HR, 1.21; 95\% CI, $1.00-1.47 ; p=0.050)$, portal vein (HR, 1.19; 95\% CI, $1.02-1.40 ; p=0.028)$, hepatic artery resections (HR, 1.62; 95\% CI, 1.06-2.49; $p=0.025$ ), positive margins (HR, 1.64; 95\% CI, 1.39-1.93, $p<0.001)$, involvement of regional lymph nodes (HR, 1.87; 95\% CI, 1.59-2.19, $p<0.001)$, presence of perineural invasion (HR, 1.66; 95\% CI, $1.32-2.08 ; p<0.001)$, and poor differentiation grade (HR, 1.39 ; 95\% CI, 1.22-1.59; $p<0.001)$. Interestingly, OS was not associated with major liver resections (HR, 0.79; 95\% CI, 0.45-1.37; $p=0.41$ ) or extended liver resections (HR, 0.82; 95\% CI, 0.47-1.43; $p=0.50$ ) (Table 2).

The Akaike information criterion (AIC) model selection was used to identify the best-fit model. In the multivariable analysis, OS was associated with positive margins (R1: HR, 1.36; 95\% CI, 1.10-1.67; $p=0.004)$, lymph node

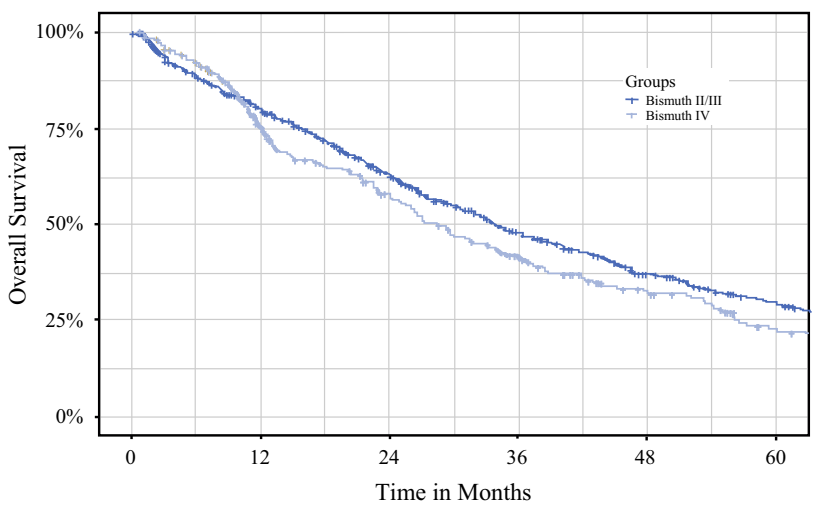

FIG. 1 Overall survival of Bismuth type 2 or 3 patients versus Bismuth type 4 patients.

metastasis (N1: HR, 1.62; 95\% CI, 1.32-1.99; $p<0.001$ ), presence of perineural invasion (HR, 1.53; 95\% CI, $1.15-2.04 ; p=0.004)$, and poorly differentiated tumor grade (HR, 1.25; 95\% CI, 1.05-1.47; $p=0.009$ ). Notably, $\mathrm{BC}$ type 4 pCCA was not associated with a poorer prognosis than BC types 2 and 3 pCCA $(p=0.063)$.

\section{Disease-Free Survival Analysis of the pCCA Patients}

In the univariable survival analysis, several factors were associated with DFS including male gender (HR, 1.30; 95\% CI, 1.05-1.61; $p=0.015)$, positive margins (R1: HR, 1.63 ; 95\% CI, 1.30-2.03; $p<0.001)$, lymph node metastasis (N1: HR, 1.86; 95\% CI, 1.51-2.30; $p<0.001$ ), extended liver resection (HR, 0.72; 95\% CI, 0.59-0.89; $p=$ 0.003 ), and poorly differentiated tumor grade (HR, 1.40; 95\% CI, 1.16-1.69; $p<0.001)$. Interestingly, major hepatectomy was not associated with DFS (HR, 1.65; 95\% CI, 0.61-4.35; $p=0.33$; Table 3).

In the multivariable Cox regression analysis, DFS was associated with male gender (HR, 1.34; 95\% CI, 1.03-1.75; $p=0.027$ ), positive margins (R1: HR, 1.46; 95\% CI, $1.12-1.90 ; p=0.005)$, lymph node metastasis (N1: HR, $1.73 ; 95 \%$ CI, 1.34-2.25; $p<0.001)$, extended liver resection (HR, $0.73 ; 95 \% \mathrm{CI}, 0.56-0.95 ; p=0.020)$, and poorly differentiated tumor grade $(\mathrm{HR}, 1.52 ; 95 \% \mathrm{CI}$, 1.21-1.90; $p<0.001)$. Notably, BC type 4 pCCA was not associated with a higher risk of recurrence than $\mathrm{BC}$ types 2 and 3 pCCA (HR, 1.00; 95\% CI, 0.80-1.26; $p=0.936$; Table 3$)$. These results were confirmed in the survival analysis for OS and DFS after a propensity score-matching (PSM) analysis among the two groups (Tables S1, S2, S3; Fig. S1). In particular, a 1:1 exact PSM was performed, identifying $306 \mathrm{BC}$ type 4 patients and 306 BC types 2 and 3 patients, with no differences in terms of preoperative clinicopathologic characteristics (all $p \geq 0.99$; Table S1). After PSM, the median LoS was 16 days (IQR, 10.0-26.0 
TABLE 2 Uni- and multivariable survival analysis: Cox's proportional hazard model for overall survival

\begin{tabular}{llllll}
\hline Variables & Univariable & & & Multivariable & \\
\cline { 2 - 2 } \cline { 5 - 6 } & HR $(95 \%$ CI $)$ & $p$ value & HR (95\% CI) & $p$ value \\
\hline Gender (male vs female) & $1.08(0.94-1.26)$ & 0.33 & & \\
Bismuth type (2 or 3 vs 4) & $0.85(0.71-1.00)$ & 0.063 & & \\
Biliary drainage (yes vs no) & $1.08(0.95-1.13)$ & 0.41 & & \\
Portal vein embolization (yes vs no) & $1.21(1.00-1.47)$ & 0.050 & & \\
Major liver resections (yes vs no) & $0.79(0.45-1.37)$ & 0.41 & & \\
Extended liver resections (yes vs no) & $0.82(0.47-1.43)$ & 0.50 & & \\
Segment 1 resected (yes vs no) & $0.97(0.79-1.20)$ & 0.81 & & \\
Portal vein resection (yes vs no) & $1.19(1.02-1.40)$ & 0.028 & & \\
Hepatic artery resection (yes vs no) & $1.62(1.06-2.49)$ & 0.025 & & \\
Margins (positive vs negative) & $1.64(1.39-1.93)$ & $<0.001$ & & $1.36(1.10-1.67)$ & 0.004 \\
N stage (N1 vs N0) & $1.87(1.59-2.19)$ & $<0.001$ & & $1.62(1.32-1.99)$ & $<0.001$ \\
Perineural invasion (yes vs no) & $1.66(1.32-2.08)$ & $<0.001$ & & $1.53(1.15-2.04)$ & 0.004 \\
Differentiation grade (poor vs well/moderately) & $1.39(1.22-1.59)$ & $<0.001$ & & $1.25(1.05-1.47)$ & 0.009 \\
\hline
\end{tabular}

HR, hazard ratio; CI, confidence interval

TABLE 3 Uni- and multivariable survival analysis: Cox's proportional hazard model for disease-free survival

\begin{tabular}{|c|c|c|c|c|}
\hline Variables & $\begin{array}{l}\text { Univariable } \\
\text { HR }(95 \% \text { CI) }\end{array}$ & $p$ value & $\begin{array}{l}\text { Multivariable } \\
\text { HR }(95 \% \text { CI) }\end{array}$ & $p$ value \\
\hline Gender (male vs female) & $1.30(1.05-1.61)$ & 0.015 & $1.34(1.03-1.75)$ & 0.027 \\
\hline Bismuth type ( 2 or 3 vs 4 ) & $1.00(0.80-1.26)$ & 0.94 & & \\
\hline Biliary drainage (yes vs no) & $0.94(0.83-1.07)$ & 0.41 & & \\
\hline Portal vein embolization (yes vs no) & $0.87(0.67-1.14)$ & 0.34 & & \\
\hline Major liver resections (yes vs no) & $1.65(0.61-4.35)$ & 0.33 & & \\
\hline Extended liver resections (yes vs no) & $0.72(0.59-0.89)$ & 0.003 & $0.73(0.56-0.95)$ & 0.020 \\
\hline Segment 1 resected (yes vs no) & $1.07(0.83-1.39)$ & 0.57 & & \\
\hline Portal vein resection (yes vs no) & $0.82(0.65-1.02)$ & 0.078 & & \\
\hline Hepatic artery resection (yes vs no) & $1.11(0.57-2.17)$ & 0.74 & & \\
\hline Margins (positive vs negative) & $1.63(1.30-2.03)$ & $<0.001$ & $1.46(1.12-1.90)$ & 0.005 \\
\hline $\mathrm{N}$ stage (N1 vs N0) & $1.86(1.51-2.30)$ & $<0.001$ & $1.73(1.34-2.25)$ & $<0.001$ \\
\hline Perineural invasion (yes vs no) & $1.32(0.98-1.78)$ & 0.059 & & \\
\hline Differentiation grade (poor vs well/moderately) & $1.40(1.16-1.69)$ & $<0.001$ & $1.52(1.21-1.90)$ & $<0.001$ \\
\hline
\end{tabular}

HR, hazard ratio; CI, confidence interval

days) for the $\mathrm{BC}$ type 4 patients compared with 12 days (IQR, 11.0-13.0 days) for the BC types 2 and 3 patients $(p<0.001)$. Moreover, compared with the BC types 2 and 3 patients, the $\mathrm{BC}$ type 4 patients had greater incidences of PHLF (PHLF B/C: BC type 4, $n=60$ [19.6\%]; BC types 2 and 3, $n=36$ [11.8\%]; $p=0.008$ ), biliary leak (biliary leak B/C: BC type 4, $n=71$ [23.2\%]; BC types 2 and 3, $n=49$ [16.1\%]; $p=0.027$ ), and severe complications (ClavienDindo $\geq 3$ : BC type 4, $n=157$ [51.3\%]; BC types 2 and 3, $n=122\{40 \%] ; p=0.005)$. Conversely the two groups had similar incidences of 30- and 90-day mortality (all $p>$ 0.13). Moreover, BC type was not associated with OS or
DFS in the uni- and multivariate analyses (all $p>0.38$; Tables S2 and S3).

\section{Prognosis of the NO BC Type 4 and BC Types 2 and 3 Patients}

For further investigation of the survival outcomes for the BC type 4 patients and BC types 2 and 3 patients, a subanalysis including only patients with no lymph node metastasis (N0) was performed. Among the N0 patients with negative surgical margins (R0), the 5-year OS rate was $43 \%$ (95\% CI, 36.7-50.3\%) for the BC types 2 and 3 
pCCA patients and $42.3 \%$ (95\% CI, 32.5-55.0\%) for the BC type 4 pCCA patients $(p=0.38)$. Among N0 patients with positive surgical margins (R1), the 5-year OS rate was $26 \%$ (95\% CI, 18.0-37.5) for the BC types 2 and 3 pCCA patients and $13.4 \%(95 \% \mathrm{CI}, 5.8-31.0)$ for the BC type 4 pCCA patients ( $p=0.40$; Fig. 2 and Fig S1).

In the Cox survival model, compared with N0/R0 BC types 2 and 3 patients, the N0/R1 BC types 2 and 3 pCCA patients and the BC type 4 pCCA patients had a $61 \%$ and $93 \%$ greater risk of death, respectively (NO/R1: BC types 2 and 3 pCCA: HR, 1.61; 95\% CI, 1.22-2.11; $p<0.001$; N0/ R1 BC type 4 pCCA: HR, 1.93; 95\% CI, 1.35-2.77; $p<$ 0.001 ), whereas the N0/R0 BC type 4 patients had a similar prognosis (N0/R0 BC type 4 pCCA: HR, 1.14; 95\% CI, $0.85-1.54 ; p=0.39)$.

\section{DISCUSSION}

Although the use of extended liver resections for pCCA has continued to increase worldwide during the last decade, BC type 4 pCCA generally has been considered a locally advanced tumor amenable to surgical treatments only for highly selected patients treated by surgeons with challenging technical skills. ${ }^{11}, 23$ Although pCCA often requires extensive surgery and a complex preoperative workup, patients with $\mathrm{BC}$ type 4 pCCA might require even a higher rate of extended hepatectomies to achieve complete resection (R0). ${ }^{24}, 25$ Reportedly, additional vascular resections, including portal vein and hepatic artery resection/ reconstruction, frequently achieve negative surgical margins (R0), increasing the complexity of surgery and resulting in higher incidences of complications and postoperative mortality. $^{24}$-26 Recently, surgical series from Asian centers have shown that good surgical outcomes can be achieved also for patients with BC type 4 pCCA,

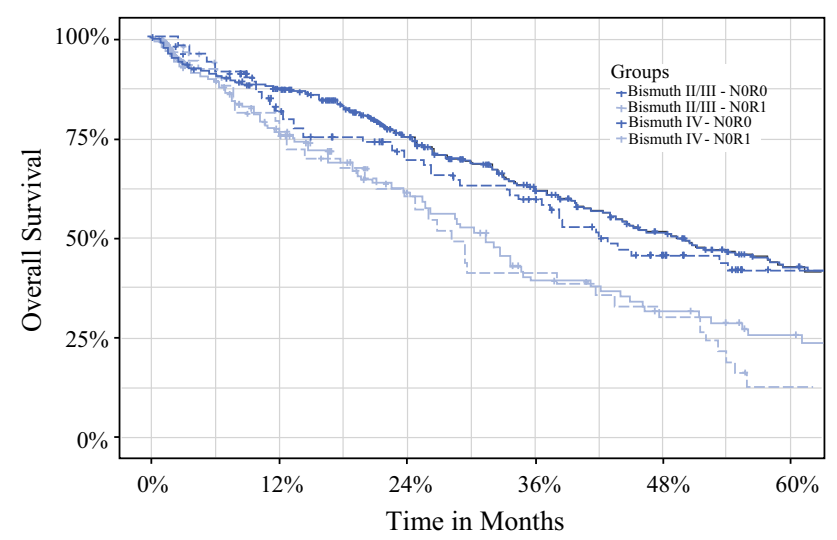

FIG. 2 Overall survival of Bismuth type 2 or 3 patients versus Bismuth type 4 NOR0 patients. providing evidence that surgical resection might offer a chance for long-term survival also for these patients with advanced tumors. ${ }^{6}, 11$

In the current study, including 1138 patients who underwent surgical resection for pCCA at 20 Western centers, surgery for BC type 4 pCCA was associated with short-term (i.e., morbidity and mortality) and long-term (i.e., OS and DFS) outcomes comparable with those achieved by surgery for BC types 2 and 3 pCCA patients. Given that the differences in baseline characteristics (e.g., rate of extended hepatectomies) between the two groups might have influenced the analysis of the short-term outcomes, especially the long-term oncologic outcomes after surgery, a 1:1 PSM was performed to mitigate measurable baseline confounding in the comparison of the BC type 4 group and the BC types 2 and 3 group. After PSM, shortterm (i.e., morbidity and mortality) and long-term (i.e., OS and DFS) outcomes were comparable between the BC type 4 pCCA patients and the BC types 2 and 3 pCCA patients.

In our analysis, the patients with $\mathrm{BC}$ type $4 \mathrm{pCCA}$ and those with $\mathrm{BC}$ types 2 and 3 pCCA demonstrated differences in terms of clinical and pathologic characteristics including incidences of PVE (BC type 4 [26\%] vs BC types 2 and 3 [18\%]; $p<0.001$ ), extended liver resection (BC type 4 [61\%] vs BC types 2 and 3 [46\%]; $p<0.001)$, and positive surgical margin (R1: BC type 4 [39\%] vs BC types 2 and 3 [31\%]; $p=0.014)$.

In comparison, Ebata et al. ${ }^{27}$ investigating the surgical outcomes for 1399 pCCA patients who underwent surgical resection at eight South-East Asian institutions reported that $\mathrm{BC}$ type 4 pCCA was associated with a higher incidence of lymph node metastasis (pN1: BC type 4 [54\%] vs BC type 3 [39\%]; $p<0.001)$ and distant metastasis (pM1: $\mathrm{BC}$ type 4 [16\%] vs BC type 3 [9\%]; $p<0,001)$ as well as a higher incidence of positive surgical margins (R1/R2: BC type 4 [37\%] vs BC type 3 [20\%]; $p<0.001)$.

Interestingly, in a recent review by $\mathrm{Ku}$ et al. ${ }^{17}$ including 292 BC type 4 pCCA patients who underwent liver surgery, the incidence of negative surgical margins (R0) ranged from $51 \%$ to $100 \%$. When only the larger case series with more than 100 patients per center were considered, the incidence of R0 resections ranged from $63 \%$ to $75 \%$ and was comparable with that of our series (R0: BC type $4[63 \%])^{27}-29$ Conversely, in our analysis, the incidence of lymph node metastases (N1) was comparable between the $\mathrm{BC}$ type 4 and $\mathrm{BC}$ types 2 and 3 pCCA patients (N1: BC type 4 [45\%] vs BC types 2 and 3 [41\%]; $p=0.23)$, probably because of more strict selection criteria adopted in the Western centers for patients with BC type 4 tumors considered for resection.

In our series, the incidence of severe complications (Clavien-Dindo $\geq 3$ ) was lower but not significantly different statistically for the $\mathrm{BC}$ types 2 and 3 patients than for 
the BC type 4 patients (complications: $\mathrm{BC}$ types 2 and 3 [46\%] vs BC type 4 [51\%]; $p=0.099)$, whereas the 90 -day mortality did not differ between the two groups (90-day mortality: BC type 4 [12\%] vs BC types 2 and 3 [13\%]; $p=$ 0.57). A 1:1 PSM analysis showed that the incidence of severe complications (Clavien-Dindo $\geq 3$ ) and 90-day mortality also were comparable between the $\mathrm{BC}$ type 4 and BC types 2 and 3 patients (BC type 4 [48\%] vs BC types 2 and 3 [46\%], $p=0.82$; 90-day mortality: $\mathrm{BC}$ type 4 [11\%] vs BC types 2 and 3 [11\%]; $p>0.99$ ).

In a recent systematic review and meta-analysis of morbidity and mortality after major liver resection for pCCA patients, Franken et al..$^{30}$ investigated the surgical outcomes for 4634 patients. Interestingly, whereas the pooled, overall incidence of severe morbidity (defined as Clavien-Dindo grade $\geq 3$ or major complications requiring surgical, endoscopic, or radiologic reintervention, and/or life-threatening complications) was $40 \% \quad(95 \%$ CI, $34-47 \%$ ) in the sub-analysis including only the Western series, the incidence of severe morbidity was $43 \%$ (95\% CI, 34-52\%), similar to our results (Clavien-Dindo $\geq 3$ complications: BC type 4: pre-PSM [51\%]; post-PSM $[48] \%){ }^{30}$ In the same review, the pooled 90 -day mortality was $9 \%(95 \% \mathrm{CI}, 6-12 \%)$ among the Western centers, comparable with the incidence of 90-day mortality (12\%; 95\% CI, 10-15\%) in our study (90-day mortality: BC type 4 patients: pre-PSM [12\%]; post-PSM [11\%]). ${ }^{30}$

Olthof et al., investigating the role of PVE in patients undergoing surgery for pCCA, found that PVE before major liver resection was associated with a lower incidence of liver failure (PVE, $8 \%$ vs no PVE, 36\%; $p<0.001$ ) and mortality (PVE, 7\% vs no PVE, $18 \% ; p=0.03$ ). These results confirm a significant difference in morbidity and mortality compared with the postoperative outcomes reported by Western and Eastern centers.

The current study confirmed that curative surgery for pCCA, even in specialized tertiary Western centers, still is associated with high complication rates. Differences in patient characteristics (i.e., incidence of obesity) and preoperative treatment approaches (i.e., incidence of preoperative biliary drainage, type of biliary drainage used, and PVE) between Western and Eastern centers might influence surgical outcomes for pCCA patients, especially for BC type 4 patients.

Recently, Kimura et al. ${ }^{32}$ compared the long- and shortterm outcomes for 183 patients who underwent radical resection for pCCA at an Eastern center (Hirosaki University Hospital, Japan) and a Western center (St. James's University Hospital, Leeds, UK). The authors reported a significant difference in the patient characteristics between the two centers. Moreover, although the 90 -day mortality rates differed $(2.5 \%$ vs $13.6 \%$, respectively), the 5-year disease-specific survival rate was $32.8 \%$ for the Eastern center and $31.9 \%$ for the Western center $(p=0.77) .^{32}$ Interestingly, in a preliminary analysis of the pCCA consortium data including three major hepatobiliary centers (Memorial Sloan Kettering Cancer Center, New York, NY; the Academic Medical Center in Amsterdam the Netherlands; Hokkaido University Graduate School of Medicine in Sapporo, Japan), the authors reported large overall differences in baseline characteristics, treatment, and clinical outcomes between an Eastern cohort (Japan) and a Western cohort (New York and Amsterdam) of patients undergoing combined hilar and liver resections for pCCA. ${ }^{33}$ Although the authors reported that the Western cohort had a worse survival and a higher 90-day mortality than the Eastern patients, after a PSM between the two cohorts, these differences were significantly attenuated.

In our analysis, the 5-year OS was $30 \%$ for the $\mathrm{BC}$ types 2 and 3 pCCA patients and $23 \%$ for the BC type 4 pCCA patients. In a recent review by $\mathrm{Ku}$ et al. ${ }^{17}$ investigating the long-term outcomes for BC type 4 pCCA patients, the OS varied considerably among the different studies examining liver resection for BC type $4 \mathrm{pCCA}$, ranging for $10.5 \%$ at 3 years to $52 \%$ at 5 years.

Ebata et al. ${ }^{11}$ investigated the oncologic outcomes of 216 patients who underwent liver resection for BC type 4 pCCA and reported a 5-year OS of 33\%, ranging from $37 \%$ for the R0 patients $(n=154)$ to $53 \%$ for the N0 patients $(n=87)$. Percutaneous biliary drainage, blood transfusion, nodal status, and distant metastasis were predictors of poor patient prognosis.

Similarly, in a multi-institutional analysis including 1352 pCCA patients who underwent surgical resection at eight Japanese centers, no differences in terms of 5-year OS among BC types 1 and 2, BC type 3, and BC type 4 N0/ R0 patients were reported (5-year OS: BC types 1 and 2 [63\%]; $\mathrm{BC}$ type 3 [66\%]; BC type 4 [59\%]; all $p>0.29$ ). $\mathrm{Li}$ et al. ${ }^{29}$ analyzing the oncologic outcomes of $142 \mathrm{BC}$ type 4 pCCA patients reported a 5 -year OS of $25 \%$ for the R0 patients and $28 \%$ for the N0 patients.

The indication for resection in $\mathrm{BC}$ type $4 \mathrm{pCCA}$ patients should be evaluated carefully in the context of the significant postoperative mortality and poor prognosis associated with negative prognostic factors (i.e., positive surgical margins, lymph-node metastasis). For these reasons, a careful selection of candidates for surgical resection and meticulous preoperative management are decisive for improvement of both the short- and long-term surgical outcomes.

In this article, we report the largest Western surgical series describing results of surgery for pCCA, emphasizing the potential benefit of surgery also for BC type 4 pCCA. Notably, promising results can be achieved for selected 
patients because in the sub-analysis for N0/R0 patients, the 5 -year OS was $43 \%$ for the BC types 2 and 3 patients and $42 \%$ for the BC type 4 pCCA patients $(p=0.38$ ).

The current study should be interpreted in light of several limitations. Although only tertiary referral centers were included in the collaborative group, given the retrospective and multi-institutional nature of the study, it was obviously not possible to standardize the surgical approaches and perioperative managements among the collaborating centers. During the study period, from 2000 to 2017, preoperative management and surgical treatments may have changed over time. Moreover, the indications for the surgical treatment of $\mathrm{BC}$ type $4 \mathrm{pCCA}$ patients were based on single-center/surgeon preferences. Importantly, in the survival analysis, we included only patients who survived at least 30 days after surgery. Although 90-day mortality might be more significant, we believe that the occurrence of very early recurrence (i.e., before 3 months) might have influenced the results of our analysis. According to these considerations, the incidence of PHLF, biliary leak, Clavien-Dindo $\geq 3$ complications, and 30-day mortality was greater among the $\mathrm{BC}$ type 4 patients than among the $\mathrm{BC}$ types 2 and 3 patients, but the difference was statistically nonsignificant. Conversely, the 90-day mortality was slightly higher among the $\mathrm{BC}$ types 2 and 3 patients $(13.4 \%)$ than among the BC type 4 patients (12.2\%), probably due to patients with a very early recurrence.

Unfortunately, due to the multicentric nature of our study, we were not able to collect the cause of death to compute the 90-day mortality for the survival analysis. Moreover, in the analysis, the impact of systemic treatment was not investigated. Although the use of adjuvant chemotherapy/radiotherapy was probably heterogeneous in the study population, further studies investigating the role of adjuvant treatments for BC type 4 pCCA patients should address this specific issue.

Finally, significant differences in terms of clinicalpathologic characteristics between the BC types 2 and 3 patients and the $\mathrm{BC}$ type 4 patients might have influenced the comparison of survival data. To mitigate the selection and confounding bias, an exact propensity score match was performed matching patients based on the most important preoperative clinicopathologic characteristics (i.e., age, sex, preoperative drainage, CA 19-9 level, preoperative $\mathrm{PVE}$, and preoperative cholangitis).

In conclusion, surgery for the selected BC type 4 pCCA patients resulted in short- and long-term outcomes similar to those for the BC types 2 and 3 pCCA patients. Although the patients in the $\mathrm{BC}$ type 4 group underwent a more "aggressive" type of surgery (i.e., vascular resection, extended hepatectomies), the incidences of severe complications and mortality were comparable with those classified as BC types 2 and 3 pCCA. Likewise, although the BC type 4 patients had worse clinical-pathologic characteristics, these patients had oncologic outcomes similar to those of the BC types 2 and 3 pCCA patients. Surgery should therefore be considered an acceptable curative treatment option for selected patients with $\mathrm{BC}$ type 4 pCCA although it requires a more technically challenging approach.

ACKNOWLEDGEMENTS Perihilar Cholangiocarcinoma Collaboration Group: Andreou A (King's College London, London, UK), Bartsch F (University Hospital of Mainz, Mainz, Germany), Benzing C (Charité University Hospital, Berlin, Germany), Buettner S (Erasmus Medical Center, Rotterdam, the Netherlands), Campagnaro T (University of Verona, Verona, Italy), Capobianco I (University Hospital Tübingen, Tübingen,Germany), Charco $\mathrm{R}$ (Universidad Autónoma de Barcelona, Barcelona, España), de Reuver P (Radboud University Medical Center, Nijmegen, The Netherlands), de Savornin Lohman E (Radboudumc, Nijmegen, The Netherlands), Dejong CHC (Maastricht University, Maastricht, The Netherlands), Efanov M (Moscow Clinical Scientific Center, Moscow, Russia), Erdmann JI (University Medical Center Groningen, Groningen, the Netherlands), Franken LC (University of Amsterdam, Amsterdam, The Netherlands), Giovinazzo G (University Hospital Aintree, Liverpool, UK), Giglio MC (Federico II University of Naples, Naples, Italy), GomezGavara C (Universidad Autónoma de Barcelona, Barcelona, España), Heid F (University Hospital of Mainz, Mainz, Germany), IJzermans JNM (University Medical Center Rotterdam, Rotterdam, The Netherlands), Isaac $\mathbf{J}$ (Queen Elizabeth Hospital Birmingham, Birmingham, United Kingdom), Jansson H (Karolinska Institutet, Stockholm, Sweden), Ligthart MAP (Rijnstate Hospital, Arnhem, The Netherlands), Maithel SK (Emory University, Atlanta, GA, USA), Malagò M (University College London, Royal Free Hospitals, London, UK), Malik HZ (Aintree University Hospital, Liverpool, UK), Muiesan P (Queen Elizabeth Hospital Birmingham, Birmingham, United Kingdom), Olde Damink SWM (Maastricht University Medical Center, Maastricht, The Netherlands), Quinn LM (University of Liverpool, Liverpool, UK), Ratti F (Ospedale San Raffaele-IRCCS, Milan, Italy), Ravaioli M (Alma Mater Studiorum - University of Bologna, Bologna, Italy), Rolinger J (University Hospital Tuebingen, Tuebingen, Germany), Schadde E (University of Zurich, Zurich, Switzerland), Serenari M (Alma Mater Studiorum - University of Bologna, Bologna, Italy), Troisi R (Federico II University of Naples, Naples, Italy), van Laarhoven S (University College London, Royal Free Hospitals, London, UK), van Vugt JLA (University Medical Center Rotterdam, Rotterdam, The Netherlands).

FUNDING Open access funding provided by Università degli Studi di Verona within the CRUI-CARE Agreement.

OPEN ACCESS This article is licensed under a Creative Commons Attribution 4.0 International License, which permits use, sharing, adaptation, distribution and reproduction in any medium or format, as long as you give appropriate credit to the original author(s) and the source, provide a link to the Creative Commons licence, and indicate if changes were made. The images or other third party material in this article are included in the article's Creative Commons licence, unless indicated otherwise in a credit line to the material. If material is not included in the article's Creative Commons licence and your intended use is not permitted by statutory regulation or exceeds the permitted use, you will need to obtain permission directly from the copyright holder. To view a copy of this licence, visit http://creativecommons. org/licenses/by/4.0/. 


\section{REFERENCES}

1. DeOliveira ML, Cunningham SC, Cameron JL, et al. Cholangiocarcinoma: thirty-one-year experience with 564 patients at a single institution. Ann Surg. 2007;245:755-62.

2. Igami T, Nishio H, Ebata T, et al. Surgical treatment of hilar cholangiocarcinoma in the "new era": the Nagoya University experience. J Hepatobiliary Pancreat Sci. 2010;17:449-54.

3. Mansour JC, Aloia TA, Crane $\mathrm{CH}$, Heimbach JK, Nagino M, Vauthey JN. Hilar cholangiocarcinoma: expert consensus statement. HPB Oxford. 2015;17:691-9.

4. Ruzzenente A, Bagante F, Ardito F, et al. Comparison of the 7th and 8th editions of the American Joint Committee on Cancer Staging Systems for perihilar cholangiocarcinoma. Surgery. 2018;164:244-50.

5. Wiggers JK, Groot Koerkamp B, Cieslak KP, et al. Postoperative mortality after liver resection for perihilar cholangiocarcinoma: development of a risk score and importance of biliary drainage of the future liver remnant. $J \mathrm{Am}$ Coll Surg. 2016;223(2):321.e1-331.e1

6. Nagino M, Ebata T, Yokoyama Y, et al. Evolution of surgical treatment for perihilar cholangiocarcinoma: a single-center 34-year review of 574 consecutive resections. Ann Surg. 2013;258:129-40.

7. Lewis HL, Rahnemai-Azar AA, Dillhoff M, Schmidt CR, Pawlik TM. Current management of perihilar cholangiocarcinoma and future perspectives. Chirurgia Bucur. 2017;112:193-207.

8. Kimbrough CW, Cloyd JM, Pawlik TM. Surgical approaches for the treatment of perihilar cholangiocarcinoma. Expert Rev Anticancer Ther. 2018;18:673-83.

9. Campagnaro T, Ruzzenente A, Bagante F, et al. Is type IV Bismuth-Corlette perihilar cholangiocarcinoma a real contraindication for curative intent surgical resection? Comparison of type Iv Vs Types I-II-III Bismuth-Corlette perihilar cholangiocarcinoma in a single tertiary referral center. Gastroenterology. 2019;156(6):S-1430.

10. Ku D, Tang R, Pang T, et al. Survival outcomes of hepatic resections in Bismuth-Corlette type IV cholangiocarcinoma. ANZ J Surg. 2020;90:1604-14.

11. Ebata T, Mizuno T, Yokoyama Y, Igami T, Sugawara G, Nagino M. Surgical resection for Bismuth type IV perihilar cholangiocarcinoma. Br J Surg. 2018;105:829-38.

12. Bagante F, Tran T, Spolverato G, et al. Perihilar cholangiocarcinoma: number of nodes examined and optimal lymph node prognostic scheme. J Am Coll Surg. 2016;222(5):750.e2-759.e2.

13. Conci S, Ruzzenente A, Sandri M, et al. What is the most accurate lymph node staging method for perihilar cholangiocarcinoma? Comparison of UICC/AJCC pN stage, number of metastatic lymph nodes, lymph node ratio, and $\log$ odds of metastatic lymph nodes. Eur J Surg Oncol. 2017;43:743-50.

14. Miyazaki M, Ohtsuka M, Miyakawa S, et al. Classification of biliary tract cancers established by the Japanese Society of Hepato-Biliary-Pancreatic Surgery. 3rd English ed. J Hepatobiliary Pancreat Sci. 2015;22:181-96.

15. van der Gaag NA, de Castro SM, Rauws EA, et al. Preoperative biliary drainage for periampullary tumors causing obstructive jaundice; DRainage vs (direct) OPeration (DROP-trial). BMC Surg. 2007;7:3.

16. Coelen RJS, Roos E, Wiggers JK, et al. Endoscopic versus percutaneous biliary drainage in patients with resectable perihilar cholangiocarcinoma: a multicentre, randomised controlled trial. Lancet Gastroenterol Hepatol. 2018;3:681-90.
17. Clavien PA, Barkun J, de Oliveira ML, et al. The Clavien-Dindo classification of surgical complications: five-year experience. Ann Surg. 2009;250:187-96.

18. Rahbari NN, Garden OJ, Padbury R, et al. Posthepatectomy liver failure: a definition and grading by the International Study Group of Liver Surgery (ISGLS). Surgery. 2011;149:713-24.

19. Koch M, Garden OJ, Padbury R, et al. Bile leakage after hepatobiliary and pancreatic surgery: a definition and grading of severity by the International Study Group of Liver Surgery. Surgery. 2011;149:680-8.

20. Rahbari NN, Garden OJ, Padbury R, et al. Post-hepatectomy haemorrhage: a definition and grading by the International Study Group of Liver Surgery (ISGLS). HPB Oxford. 2011;13:528-35.

21. Austin PC. Balance diagnostics for comparing the distribution of baseline covariates between treatment groups in propensity-score matched samples. Stat Med. 2009;28(25):3083-107. https://doi. org/10.1002/sim.3697.

22. Sekhon JS, Grieve RD. A matching method for improving covariate balance in cost-effectiveness analyses. Health Econ. 2012;21:695-714.

23. Govil S, Reddy MS, Rela M. Surgical resection techniques for locally advanced hilar cholangiocarcinoma. Langenbecks Arch Surg. 2014;399:707-16.

24. Matsukuma S, Tokumitsu Y, Shindo Y, Matsui H, Nagano H. Essential updates to the surgical treatment of biliary tract cancer. Ann Gastroenterol Surg. 2019;3:378-89.

25. Mizuno T, Ebata T, Nagino M. Advanced hilar cholangiocarcinoma: an aggressive surgical approach for the treatment of advanced hilar cholangiocarcinoma: perioperative management, extended procedures, and multidisciplinary approaches. Surg Oncol. 2020;33:201-6.

26. Bagante F, Ruzzenente A, Beal EW, et al. Complications after liver surgery: a benchmark analysis. HPB Oxford. 2019;21:1139-49.

27. Ebata T, Kosuge T, Hirano S, et al. Proposal to modify the International Union Against Cancer staging system for perihilar cholangiocarcinomas. Br J Surg. 2014;101:79-88.

28. Ku D, Tang R, Pang T, et al. Survival outcomes of hepatic resections in Bismuth-Corlette type IV cholangiocarcinoma. ANZ J Surg. 2020;90(9):1604-14.

29. Li B, Xiong XZ, Zhou Y, et al. Prognostic value of lymphovascular invasion in Bismuth-Corlette type IV hilar cholangiocarcinoma. World J Gastroenterol. 2017;23:6685-93.

30. Franken LC, Schreuder AM, Roos E, et al. Morbidity and mortality after major liver resection in patients with perihilar cholangiocarcinoma: a systematic review and meta-analysis. Surgery. 2019;165:918-28.

31. Olthof PB, Aldrighetti L, Alikhanov R, et al. Portal vein embolization is associated with reduced liver failure and mortality in high-risk resections for perihilar cholangiocarcinoma. Ann Surg Oncol. 2020;27(7):2311-18.

32. Kimura N, Young AL, Toyoki Y, et al. Radical operation for hilar cholangiocarcinoma in comparable Eastern and Western centers: outcome analysis and prognostic factors. Surgery. 2017;162:500-14.

33. Olthof PB, Miyasaka M, Koerkamp BG, et al. A comparison of treatment and outcomes of perihilar cholangiocarcinoma between Eastern and Western centers. HPB Oxford. 2019;21:345-51.

Publisher's Note Springer Nature remains neutral with regard to jurisdictional claims in published maps and institutional affiliations. 